\title{
Side Flow Stabilization of a Stepped-Nose Obstacle (Experimental Documentation)
}

\author{
By Anang CAKrawala and Akira Umemura \\ Department of Aerospace Engineering, Graduate School of Engineering, Nagoya University, Nagoya, Japan
}

(Received October 7th, 2002)

\begin{abstract}
A stepped-nose with various step lengths and heights, attached to a square cylinder, can significantly reduce the drag coefficient compared to that of the square cylinder. The underlying physics are that (1) the vortices trapped in the step regions produce the thrust forces acting on the step surfaces facing against the uniform stream which cancel the drag force acting on the front surface of the stepped-nose obstacle, and (2) the tangent reattachment of the flow separating from the front surface edges to the side surfaces of the main body decreases the suction pressure acting on the back surface of the main body. In the present study, these favorable effects of the stepped-nose are experimentally documented by presenting the measurement results of surface pressure coefficient, streamwise velocity, and turbulence intensity of side flow and flow visualization pictures. It is demonstrated that when step height takes a value of about one-tenth of the main body length, there is a rather wide range of step length, for which the net drag force acting on the stepped-nose almost vanishes and the side flow is stabilized by the stepped-nose.
\end{abstract}

Key Words: Stepped-Nose Obstacle, Drag Reduction, Separation Control, Wind Tunnel Experiment

\author{
Nomenclature \\ $h$ : step height \\ $H$ : main body height \\ $\ell$ : step length \\ $L$ : main body length \\ $\mathrm{s}$ : center of obstacle back surface \\ $T u$ : turbulence intensity \\ $u^{\prime}$ : streamwise fluctuating velocity \\ $U$ : mainstream velocity \\ $v^{\prime}$ : lateral fluctuating velocity \\ $\overline{u v}$ : Reynolds stress \\ Superscript/subscript \\ $\infty$ : value in free-stream state \\ bottom: value at position closest to obstacle surface \\ edge: value at outer edge \\ lateral: value for lateral direction \\ max: value at maximum \\ mean: value at time-averaged \\ streamwise: value for stream-wise direction \\ rms: value at root mean square
}

\section{Introduction}

Separated flows, typical of flows around bluff bodies, occur in a large variety of environmental and engineering situations. Flow separation has a significant impact on the performance of airfoils, turbine blades, diffusers, heat exchangers, combustors, and so on. Separated flows determine the drag of road vehicles and are the dominant feature of atmospheric flows over buildings, fences, and hills. They are also critical factors in the designing of structures, such as

(C) 2003 The Japan Society for Aeronautical and Space Sciences bridges, which are susceptible to potentially disastrous wind. Therefore, there is much research conducted to reduce the drag forces of blunt bodies. Among them, the use of a stepped-nose is a method that treats such problems by controlling flow separation, turbulence intensities, and boundary layer growth at the sidewall of a bluff body. The method may be regarded as a variation of the drag reduction method studied by Saunders ${ }^{1)}$ and Koenig and Roshko, ${ }^{2)}$ who experimented with a flat-faced, circular cylinder shielded by a disk placed coaxially upstream and demonstrated remarkable drag reduction for certain diameter and gap ratios such that the stream surface, which separates from the disk, reattaches smoothly onto the front edge of the cylinder. Watanabe $^{3)}$ showed that a great reduction of pressure drag acting on the flat head of a circular cylinder with its axis parallel to a uniform stream was achieved by notching the perimeter of the flat head into a step form. In our previous study, ${ }^{4)}$ we found, in the theoretical exploration of the underlying physics missed in these previous experimental studies, that for an appropriate choice of step sizes, the flow separated from both edges of the front surface of a steppednose obstacle reattaches just at the leading edges of the side surfaces, so that the flow separation from the obstacle sides is avoided, thus leading to a significant reduction in net drag force. The method used in this study was based on the numerical calculation of unsteady flow past a stepped-nose obstacle with a square main body and theoretical consideration.

The stepped-nose drag reduction method is especially useful for short obstacles, such as a square obstacle, which have large-scale flow separation and experience relatively large drag coefficients.

An experimental investigation of a turbulent shear flow with separation, reverse flow, and reattachment was con- 
ducted by Ruderich and Fernholz. ${ }^{5)}$ The flow investigated in that paper was created by a thin laminar boundary layer on a bluff plate that separated at the sharp beveled edge, formed a curved mixing layer-bounded on its lower side by the reverse flow region and reattached onto the splitter plate. It was concluded that there was no flapping of the reattaching shear layer. Then other researchers, Ota et al., ${ }^{6}$ investigated the characteristics of the reattachment length of a squaresection model. It is clear that the reattachment length for a square obstacle, at any Reynolds number, is more than the length of the obstacle itself. The stepped-nose method considered in this study is expected to be useful for a short square cylinder.

The most important finding from our numerical and theoretical investigation is that the step configuration, which is effective to reduce the drag, is such that makes the separated flow reattach smoothly to the leading edges of the side surfaces of the main body. According to the inviscid flow prediction, this configuration is uniquely determined. However, the viscosity extends the range of the step size, which acts in the same way as the optimum configuration and increases the applicability of this drag reduction method in engineering problems. Thus, we are conducting a series of experiments to validate the theoretical predictions. Such validation is necessary to apply the drag reduction method to practical bodies because the current simulation code is not complete in the terms of description of turbulence, which is not avoidable in a real flow situation. This paper summarizes a detailed experimental investigation on flow properties of a separated shear layer at the side surface of a stepped-nose obstacle.

\section{Experimental Apparatus and Procedure}

\subsection{Flow parameter and model dimensions}

The experiments were conducted in a $45 \mathrm{~cm} \times 34 \mathrm{~cm}$ wind tunnel at Nagoya University's Aerospace Engineering Department in the Propulsion Energy Systems Laboratory. Figure 1(a) illustrates the construction of the two-dimensional stepped-nose obstacle installed in the test section. A block with a square cross-section is partially inserted into a casing that constitutes the square form of the main body of the stepped-nose obstacle. The size of the main body is $50 \mathrm{~mm}$ in height and $50 \mathrm{~mm}$ in length. The step height $(h)$ is determined by the casing thickness, while the step length $(\ell)$ can be varied by replacing the insertion part. As shown in Fig. 1(b), the stepped-nose obstacle is mounted at the center of the test section, which is located about $5 H$ downstream of the end of the wind tunnel contraction. The solid blockage of the stepped-nose obstacles used in the present experiment was about $10 \%$. The value of the Reynolds number based on the obstacle height $(H=50 \mathrm{~mm})$ and free-stream flow velocity $\left(U_{\infty}=10 \mathrm{~m} / \mathrm{s}\right)$ is about $5.0 \times 10^{4}$. To examine Reynolds number effects, the Reynolds number was changed by increasing the free-stream velocity of the wind tunnel. The maximum Reynolds number was limited by the wind tunnel operating envelope, which has a maximum

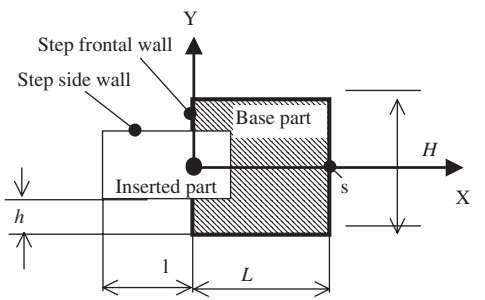

(a)

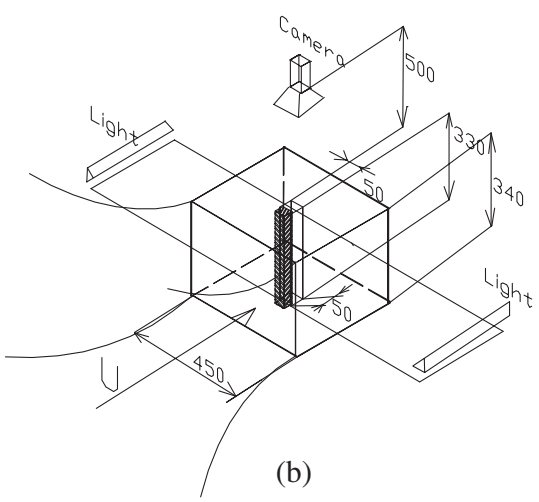

Fig. 1. Experimental setup and model description.

of about $32 \mathrm{~m} / \mathrm{s}$. We believe that this experiment is sufficient to measure the stepped-nose effect on side flow properties in the two-dimensional flow around a stepped-nose obstacle. All quantities were made dimensionless. The length, velocity, and time were made dimensionless using the obstacle height $H$ and uniform velocity $U_{\infty}$.

\subsection{Pressure measurement}

The static pressure along the body surface was measured by means of pressure transducers. Pressure orifices with $0.5 \mathrm{~mm}$ diameters were drilled in the casing and inserting block to measure the surface static pressure distribution at the mid-span of the obstacle beam. The main body part was outfitted with 28 pressure orifices and the inserted block with 6 to 18 pressure orifices. In order to minimize interference effects between pressure orifices, the pressure orifices were arranged inclined to the uniform stream. To prevent the time lag difference of each orifice measurement, vinyl tubes of equal length were used to connect the transducer to the orifices on the obstacle. The static pressure distribution on the surface of the stepped-nose obstacle was measured using differential pressure transducers and a 48-port scanivalve system. The pressure measured at the test section inlet was used as reference static and dynamic pressure, thus we could calculate the test section free-stream velocity. The reference static pressure was also used as reference static pressure for the scanivalve pressure measuring system. By using the calibrated result, we could know the measured pressure and then calculate the velocity of each measuring point. In this experiment, to determine the pressure average, we used a time-averaging pressure measurement system applying an integration time of about $2 \mathrm{~s}$, and data acquisition frequency of about $5,000 \mathrm{~Hz}$ resulted in the total number of pressure data being 10,480 .

In a recent investigation, the pressure distribution in sev- 
eral of step lengths $(\ell)$ was obtained by replacing the inserted model. Moreover, the location of pressure orifices on both sides of the obstacle was manufactured asymmetrically. Therefore, in the case of large step length, the pressure distribution displayed seemed to have a different profile. However, the high repeatability and accuracy of the pressure measurement was confirmed by using different pressure measurements.

\subsection{Velocity field measurement}

An X-probe hotwire anemometer was used to measure the flow field velocity component, turbulence intensity characteristics, and Reynolds stress distributions on the side of the obstacle. The hotwire apparatus consisted of a Kanomax $\mathrm{X}$-probe hotwire anemometer and Sokken hotwire flow meter model HC-30. The continuous time signals from the hotwire were digitized by an interface $\mathrm{AD}$ converter through a computer PCI board. A mechanical traverse system was used to move the probe across the side flow. It was selected based on the size of the area to be traversed, positioning accuracy, and anticipated fluid dynamic force acting on the traverse. Having selected the PC controlled traverse system, hotwire calibration was carried out in order to make sure that the motion of the traverse and the acquisition of data could be timed securely.

\subsection{Turbulence intensity measurement}

Turbulence levels in this measurement were very high where the flow is separated from the obstacle. We applied a correction method for the turbulence intensity measurement as used by Fernholz and Vagt ${ }^{7)}$ in the turbulence measurement of an adverse pressure gradient three-dimensional turbulence boundary layer along a circular cylinder. In the experiment, time-averaged turbulence measurement was used to measure the turbulence intensities around the obstacle. The behaviors of oscillating shear layer appearing on the side surfaces of the obstacle were considered. This was a compromise method for both accuracy and analysis time. ${ }^{8)}$ Thus, hotwire anemometer measurement was conducted at fixed sampling time $t=2.048 \mathrm{~s}$ and sampling frequency $f=1000 \mathrm{~Hz}$. The total number of data points at each measuring point was 2048. Streamwise (parallel to the side surfaces of the obstacle) and lateral (normal to the side surfaces of the obstacle) turbulence intensities in a low-speed wind tunnel with a two-dimensional steppednose obstacle are major contributors to total turbulence intensities. In this experiment, turbulence intensity measurement was limited to streamwise and lateral fluctuation velocities, $u^{\prime}$ and $v^{\prime}$. The total turbulence intensity, $T u$, was calculated from

$T u=\left(\frac{u_{\mathrm{rms}}^{\prime}}{U_{\text {mean }}}\right)=\sqrt{\frac{1}{2}\left[\left(\frac{u^{\prime}}{U_{\text {mean }}}\right)_{\text {streamwise }}+\left(\frac{v^{\prime}}{U_{\text {mean }}}\right)_{\text {lateral }}\right]}$,

where $u_{\mathrm{rms}}^{\prime}$ denotes root mean square of fluctuation velocity and $U_{\text {mean }}$ denotes mean time of stream velocity. In order to make a general comparison in general of turbulence intensity distribution, $T u$ is magnified by 100 times in graphical display, giving the percentage of the turbulence intensity at each measuring point. Then, the mean velocity was estimated from a discrete time history as the average over one polar measurement. One polar means a velocity measurement at one measuring point for a certain time.

$$
U_{\text {mean }}=\frac{1}{N} \sum_{1}^{N} U_{i}
$$

In order to calculate the turbulence intensities of the shear layer flow, we used the standard deviation of velocity as shown in the following equation

$$
U_{\mathrm{rms}}=\sqrt{\frac{1}{N} \sum_{1}^{N}\left(U_{i}-U_{\text {mean }}\right)^{2}}
$$

where $N$ is the total number of data points at each location and $U_{i}$ is the measured value.

The low-speed wind tunnel used in this experiment had a turbulence reduction system or flow conditioning that consisting of a honeycomb and three screens located upstream of the contraction. The elements were arranged to provide sufficient attenuation of turbulence intensities, thus the free-stream turbulence intensity was about $2 \%$.

The classic way of dealing with turbulence in the equation of motion is Reynolds shear stress. We used the following equation to determine the Reynolds stress of the shear layer.

$$
\overline{u v}=\frac{1}{N} \sum_{1}^{N}\left(U_{i}-U_{\text {mean }}\right) \cdot\left(V_{i}-V_{\text {mean }}\right)
$$

\section{Results and Discussion}

\subsection{Flow visualization}

The flow around an obstacle was visualized with the smoke method. In this visualization experiment, the Reynolds number of the uniform stream was reduced to $2.5 \times$ $10^{4}$ to take clear images. Figure 2 shows several pictures taken in this experiment. For comparison, the flows past square and circular cylinders were also visualized. Since the light sheet incident at the mid-span station of the obstacle beam is scattered by smoke particles, the bright portions in the photos show where there are smoke particles convected by the uniform stream. Thus, the dark portions in the pictures correspond to the separated flow regions. Considering the temporal change in the outer edges of the separated flow regions and the entrainment of smoke particles by vortices, the photos are considered to show time-averaged flow patterns. There are parallax effects on the pictures in Fig. 2. The cross-sectional area of the obstacle beam at the light sheet station must be smaller than that shown on the pictures, since the camera was located $60 \mathrm{~cm}$ away from the beam end on the centerline of the beam (see Fig. 1). The white line drawn in the image of the beam-end surface expresses the cross section of the obstacle beam at the light sheet station.

We performed a series of flow visualization experiments for cases of fixed step length $(\ell=0.16)$ with various step 

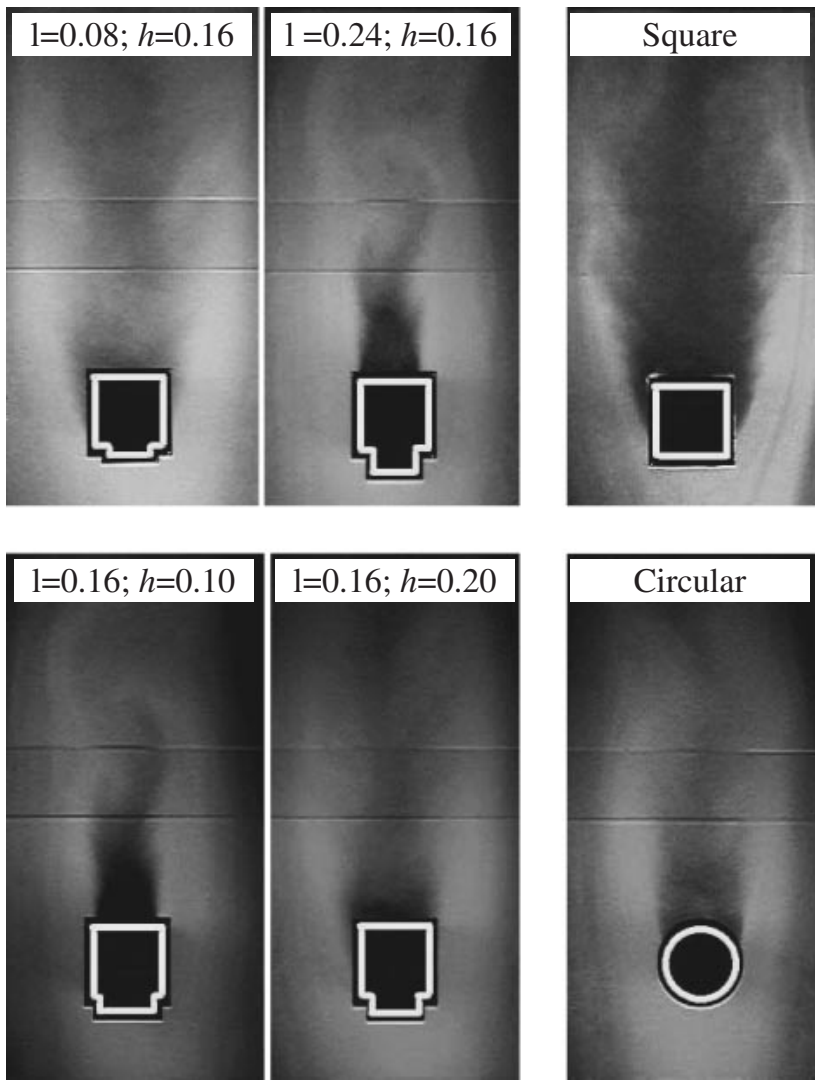

Fig. 2. Flow visualization by using smoked method.

heights, and fixed step height $(h=0.16)$ with various step lengths. As seen in Fig. 2, there is similarity between the two cases $(\ell=0.16, h=0.1)$ and $(\ell=0.24, h=0.16)$. The width of wake for these two cases is smaller than that for the circular cylinder. On the other hand, the stepped-nose obstacles with $(\ell=0.16, h=0.2)$ and $(\ell=0.08, h=0.16)$ have the same width of wake as the square cylinder, for which the main flow totally separates from the front surface edges. Therefore, it is obvious that the appropriate step-size choice suppresses the large-scale flow separation on both sides of the stepped-nose obstacle and leads to a great drag reduction compared to the square and circular cylinder cases.

\subsection{Step-length effect on side flow}

\subsubsection{Surface pressure coefficient}

In most practical situations, three-dimensional flow-fields are the rule rather than the exception. The analysis of the complex character of three-dimensional separated shear layers is a difficult task, and therefore most investigations are concentrating on two-dimensional configurations. As describe in the paper by Ruderich and Fernholz, ${ }^{5)}$ the effect of side plates and aspect ratio on the two-dimensionality of the flow configuration of forward facing step was investigated. They found that the flow was nominally two-dimensional if the aspect ratio was larger than 10 where the aspect ratio is formed by tunnel and height of the step. With the present experiment configuration, the ratio of tunnel width and step height is more than 10 , which is sufficient to maintain two-dimensional flow. However, there is a strong three-
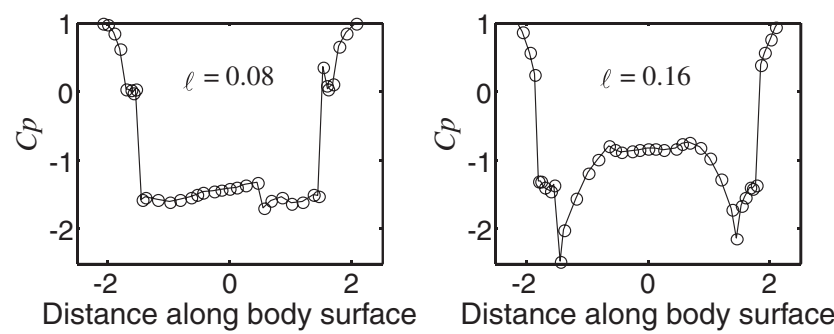

Distance along body surface
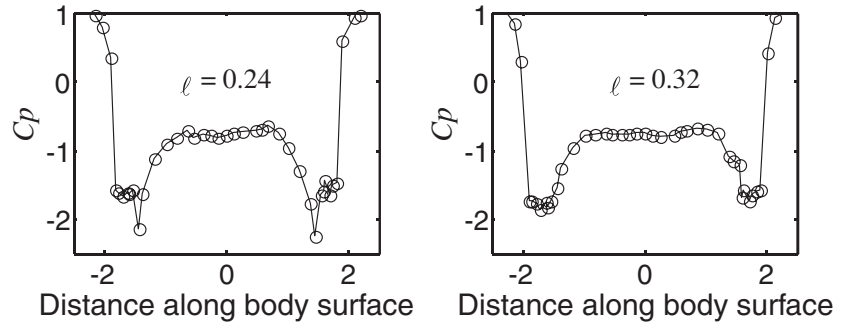

Fig. 3. Surface pressure distribution.

dimensional flow effect that occurs at the top and bottom of the obstacle due to boundary layer growth along the wind tunnel wall. In order to minimize the three-dimensional flow effect during pressure measurement, the pressure orifice was located around mid-span of the obstacle, which was located in vicinity of the center of the test section. Therefore, we assumed the flow around the obstacle for this experiment is two-dimensional flow, and this was confirmed by flow visualization results.

The time-averaged surface pressure distribution was measured for various step lengths at a fixed step height $h=0.16$. The results are shown in Fig. 3 , which indicates that the surface pressure distribution is significantly influenced by the step size. The origin of the abscissa, $s$ (refer to Fig. 1), expresses the center of the obstacle's back surface, and the distance measured from this point along the perimeter of the obstacle's cross section toward the center of the front surface is non-dimensionalized by the length of the main body. The change in step length not only affects the step region flow, but also the overall flow features around the obstacle, so that the pressure coefficient at the center of the obstacle's back surface increases from -1.2 to -0.6 (the suction pressure acting at back surface pressure decreases) with increasing step length. Observation of the pressure distribution on both sides of the main body $(0.5<s<1.5$ and $-0.5>s>-1.5)$ reveals that the dependence of side surface pressure on step length is different between the two cases: $\ell<0.16$ and $\ell>0.16$. In the short step-length case $(\ell<0.16)$, the value of the time-averaged pressure coefficient is almost unchanged along either side surface of the main body. This implies that the main flow is completely separated on both sides of the main body, as confirmed by the visualization picture in Fig. 2. On the other hand, in the long step-length case $(\ell>0.16)$, the value of the time-averaged pressure coefficient gradually increases downstream along either side surface of the step obstacle, implying that the flow attaches to the side surfaces. This can be also confirmed in Fig. 2. Thus, it is concluded that step length $\ell>0.24$ prevents large-scale flow separation 


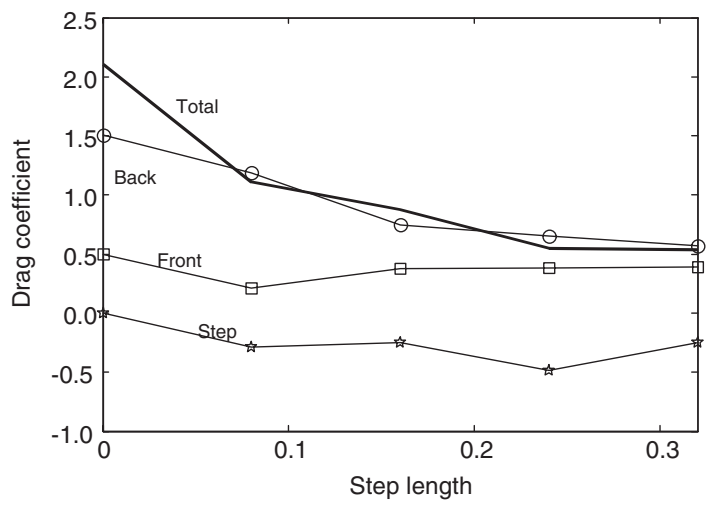

Fig. 4. Dependence of drag coefficient on step length.

on the side surfaces of the obstacle. The surface pressure distribution in the case of step length $\ell=0.16$ shows a similar profile to measurements for other longer step lengths, in that pressure relaxation occurs on the side surface of the obstacle. However, the off-body measurement such as turbulence intensity and velocity profile (presented later) shows a difference from the other step lengths, implying that small-scale separation occurs on the step length $\ell=0.16$ that causes high turbulence intensity. Thus, to distinguish the differences of the stepped-nose characteristics here, we mainly used a measurement results of the stepped-nose obstacle with step lengths $\ell=0.08$ and $\ell=0.24$.

As shown in Fig. 3, the peak of the pressure coefficient around $|s|= \pm 1.5$ looks like a different profile. The main reason is that the step-region pressure distribution is not uniform. This is caused by surface imperfection around pressure orifice or an error on zero alignment of the obstacle.

\subsubsection{Drag coefficient}

The drag coefficient of the stepped-nose obstacle is obtained by integrating the pressure coefficient over the surfaces facing normal to the uniform stream. Figure 4 denotes the dependence of drag coefficient on step length. The thick solid line denotes the total drag coefficient and other thin solid lines show the contributions of surface parts as labeled in the figure. The value of the drag coefficient for the square cylinder (i.e., $\ell=0$ ) measured in this experiment is 2.2, which is consistent with the previously reported values (2.04 at $R e=1.76 \times 10^{5}$ in Lee experiment, ${ }^{9)} 2.15$ at $R e=$ $1.3 \times 10^{4}$ in Norberg experiment, ${ }^{10)} 2.09$ at $1.0 \times 10^{5}$ in Murakami and Machida 2D CFD, ${ }^{11)} 2.37$ at $R e=1.4 \times$ $10^{4}$ in Koutmos and Mavridis 2D CFD ${ }^{12)}$ ). Furthermore, the dependencies of aerodynamic characteristics on step length, shown in Fig. 4, are qualitatively in good agreement with our numerical results. Specifically, the drag coefficient of the stepped-nose obstacles reduced to half the value of the simple square obstacle as the step length is increased. There is no significant difference between the total drag coefficient and the back surface drag coefficient contribution, which means that the net drag coefficient of the forebody of the obstacle become zero as step length increases to more than 0.24 . It is concluded that the stepped-nose is effective for reducing the drag of the obstacle when the net drag of the stepped-nose part becomes zero, and the total drag acting on the

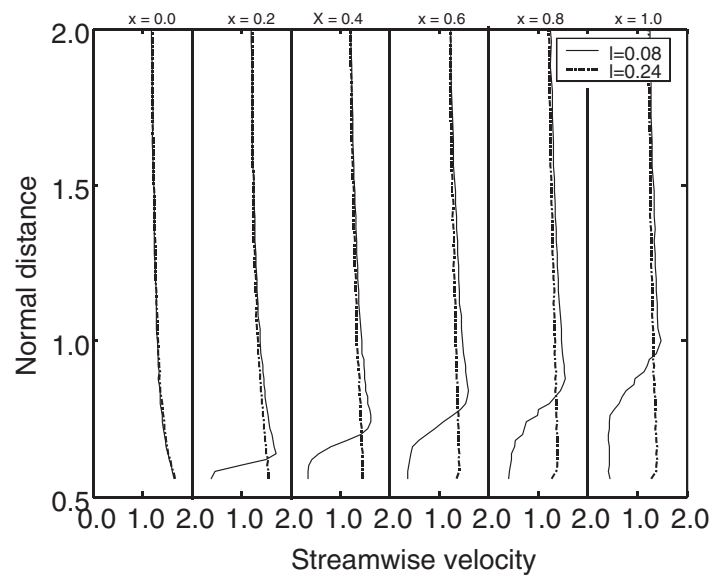

Fig. 5. Streamwise velocity distribution of side flow in cases of $h=0.16$, $\ell=0.08$, and 0.24 .

obstacle is represented by the drag acting on the back surface of the obstacle.

\subsubsection{Velocity distribution}

In order to characterize the flow behavior on the side surface downstream of the stepped-nose obstacle with various step lengths, the variations on time-averaged streamwise velocity along the side surface of the stepped-nose obstacle, defined at five stations $(x=0.0,0.2,0.4,0.6,0.8$, and 1.0 ), were measured with a $X$-probe hotwire anemometer. As described in the previous section, the X-probe hotwire anemometer has a limitation to velocity measurement in the vicinity of the side surface due to the probe support size, which is $8 \mathrm{~mm}$ in diameter. The closest measuring position is $4 \mathrm{~mm}$ from the side surface of the obstacle.

In Fig. 5, the velocity profiles are shown for the cases of $\ell=0.08$ (less drag reduction) and $\ell=0.24$ (greater drag reduction). The differences observed in Fig. 5 are mainly attributed to the effect of step length. The separated shear layer growth in the case of $\ell=0.08$ is faster than the case of $\ell=0.24$ due to flow re-separation at the leading edges of the side surfaces of the stepped-nose obstacle. On the other hand, in the case of $\ell=0.24$, the flow becomes reattached just at the leading edge of the side surface. This means that the velocity at the closest measuring position to the side surface (called "surface flow" hereafter) gradually decreases downstream in the course of pressure relaxation (see Fig. 3). In order to characterize these velocity profiles for various step lengths, the velocity values at some representative positions are employed. We use the subscript bottom to denote the closest measuring point to the side surface, max to denote the maximum velocity value at the measuring stations, and edge to denote the measuring position $y=3.5$, quite far apart from the side surface. As seen in Fig. 6, the surface velocity $U_{\text {bottom }}$ initially increases, maximizes at $\ell=0.16$, and then decreases to $U_{\text {bottom }}=1.4$ as step length increases. The surface velocity $U_{\text {bottom }}$ at each station has wide variations due to the formation of recirculating flow on the side surfaces for short step lengths. As seen in Fig. 6, the maximum velocity $U_{\max }$ has no significant difference for each measuring station, especially for step lengths 


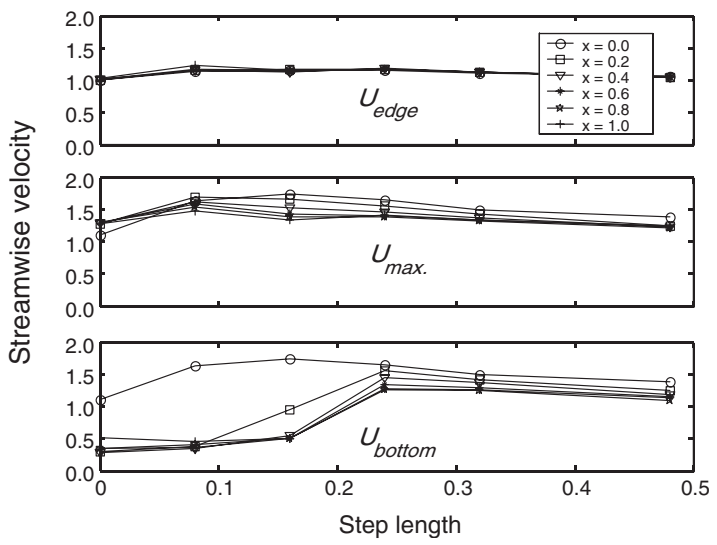

Fig. 6. Characterization of step-length dependence of time-averaged side flow for $h=0.16$.

of $\ell=0.24$ or more. The dependency of maximum velocity on step length can be identified when the behavior of separated flow discussed in the previous sections is taken into account. As we know, the maximum velocity is caused by flow acceleration due to solid blockage of the obstacle and flow separation. Although the solid blockage generally changes depending on the shape of the obstacle, the solid blockage relevant in this experiment was not significantly affected by the increase in step length. Thus, in the short step lengths, we assume that the wake blockage increases due to largescale flow separation at the side surfaces. However, in the long step-length case, large-scale flow separation is prevented by the stepped-nose. As a result, the wake blockage is almost the same and the maximum value is nearly unchanged with step length.

Although we did not measure points others than those marked in Fig. 5, we assumed that the thin boundary layer grows in the case of $\ell=0.24$, especially $x<0.4$, considering that the boundary layer growth on the side surface is very thin for step length $\ell=0.24$. Therefore, the velocity profiles are sufficient to present the boundary layer profile of the stepped-nose obstacle. The curvature of the velocity profile has a fundamental effect on stability of boundary layer. As shown in Fig. 5, it is clear that the boundary layer growth in the case of $\ell=0.24$ is more stable than that in the case of $\ell=0.08$.

A comparison of the maximum velocity $U_{\max }$ and surface velocity $U_{\text {bottom }}$ at various stations reveals the stability nature of the side flow. It was observed that, in the case of $\ell>0.24, U_{\max }$ takes a close value to $U_{\text {bottom }}$ at any station, indicating that the boundary layer thickness is very thin and the boundary layer developed along the side surface is stable. On the other hand, in the case of $\ell<0.24$, the maximum velocity $U_{\max }$ is quite different from the surface velocity $U_{\text {bottom }}$ except at $x=0$. This means that the main flow reseparates on the side surfaces immediately downstream of their leading edges, forming large-scale circulation regions along the side surface. It is considered that, in the case of short step length, the flow separating from the front surface edges impinges on the step's frontal wall and then re-separates near the leading edges of the side surfaces.

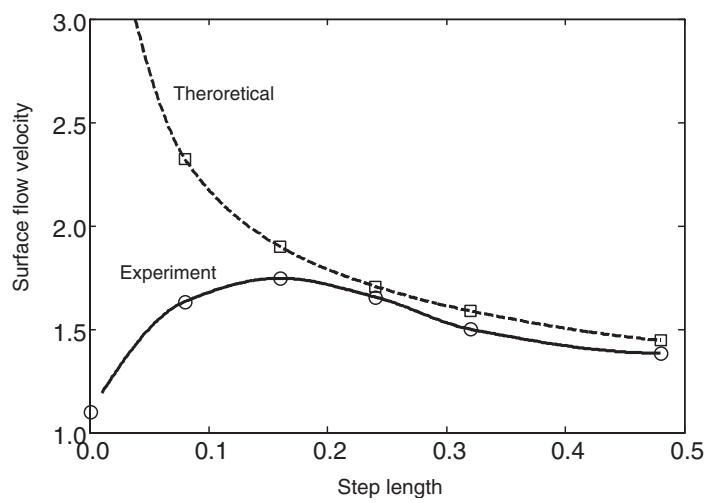

Fig. 7. Step length dependency of surface velocity at the leading edge of the side surface.

The edge velocity $U_{\text {edge }}$ is not affected by step length, but takes an approximately $10 \%$ greater value than the freestream velocity due to the blockage effect of the obstacle in the test section.

It is interesting to compare the measured surface velocity at the leading edges of the side surfaces $(x=0)$ to that predicted from an inviscid flow theory. In our previous paper, the potential flow past a semi-infinitely long body with a stepped-nose was solved in the framework of discontinuous flow theory assuming that the flow separating from the front surface edges attaches tangentially to the side surfaces of the semi-infinitely long main body at their leading edges. The fluid enclosed by these streamlines of separated flow and step surfaces is assumed stagnant, so that the speed of the fluid particles is constant everywhere on the separated flow streamlines. In particular, the value must be consistent with the surface velocity at the leading edges of the side surfaces. The broken line in Fig. 7 shows the dependence of the theoretically predicted surface velocity on step length (note that the discontinuous flow theory determines the step height as a function of step length), which should be compared to the measured surface velocity drawn as the solid line. Figure 7 shows that the two lines are in good agreement for step lengths exceeding 0.24. This suggests that the main flow of a long step length becomes similar to the discontinuous flow. The measured surface velocity is slightly lower than the theoretically predicted values because of viscous effects. The discrepancy between the two lines is significantly enlarged as the step length is decreased from $\ell=0.24$ because the main flow separates from the side surfaces of a steppednose obstacle with short step length.

As seen in Fig. 3, the pressure coefficient distribution in the stepped-nose regions varies significantly $\left(0.1>C_{P}>\right.$ -1.8 ) with increasing step length. The contribution of suction pressure acting at the step's frontal wall to the drag coefficient depends on the strength of vortices trapped in the step regions. The step configuration becomes optimum when the vortices trapped in the step regions are strong enough to lead to the vanishing net forebody drag. This condition is satisfied at step length $\ell=0.24$ as mentioned in the previous section, and the flow separating from the front surface edges smoothly reattaches to the leading edges of the 


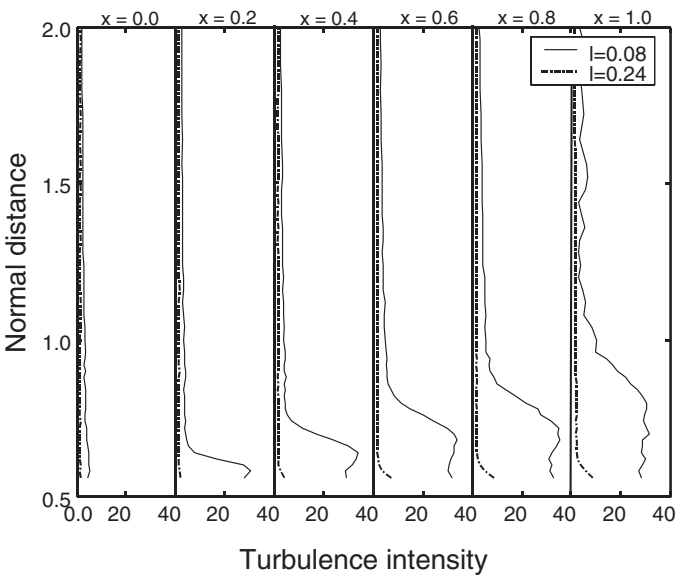

Fig. 8. Turbulence intensity distribution of side flow in cases of $h=0.16$, $\ell=0.08$, and 0.24 .

side surfaces of the main body. Importantly, even in the case of $\ell>0.24$, for which the suction pressure gradually become weak, the vortices trapped in the step regions can continue to adjust the reattachment position of the separated flow in similar way to the optimum step configuration $\ell=$ 0.24 . Thus, the surface pressure distribution of $\ell>0.24$ is similar to that of $\ell=0.24$, consistent with the theoretical prediction made in our previous paper. ${ }^{4)}$ The stabilization effect of side flow over a wide range of step lengths extends the applicability of the stepped-nose drag reduction method to practical problems.

\subsubsection{Turbulence intensity}

Figure 8 shows the distribution of turbulence intensities at various measuring positions for various step lengths. The case $\ell=0.08$ results in high turbulence, whereas the case $\ell=0.24$ yields low turbulence. These properties are consistent with the side flow behaviors described in the previous sections. The high turbulence at $\ell=0.08$ is caused by the velocity fluctuation about the time-averaged profile shown in Fig. 5 due to an oscillatory change in side flow separation to cope with the Karman vortices.

Generally, the main contributor to turbulence intensity for edge turbulence is the free-stream turbulence remaining from the turbulence reduction system (screens, honeycomb, etc.) located upstream of the test section. Turbulence intensity around the stepped-nose obstacle in this experiment was contributed by free-stream turbulence, separated flow, and disturbances from the wake of the obstacle. It is important to note that the free-stream turbulence $T u_{\infty}$ in the wind tunnel used in this experiment is about $2 \%$. In the case of a short step length, high turbulence was observed, which confirms the occurrence of large-scale flow separation at the side surfaces of the obstacles. On the other hand, the low turbulence observed in the long step-length case means that there is no large-scale separation on the side surfaces of obstacles with long step lengths, consistent with the flow visualization. As seen in Fig. 8, for the long-step length case $\ell \geq 0.24$, the turbulence intensity near the side surfaces has almost the same value as the free-stream turbulence intensity.

Figure 9 shows the characterization of turbulence intensi-

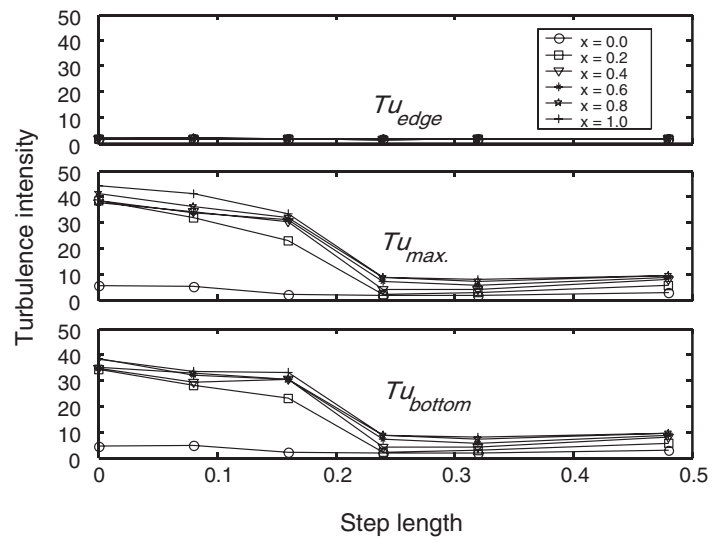

Fig. 9. Characterization of step-length dependence of turbulence intensity for $h=0.16$.

ty for various step lengths. The surface turbulence $T u_{\text {bottom }}$ denotes turbulence intensity at the measuring position closest to the side surface, $T u_{\max }$ denotes the maximum turbulence intensity at measuring stations, and edge turbulence $T u_{\text {edge }}$ denotes the turbulence intensity at the outer edge of the measuring station $(y=3.5)$. The large values of $T u_{\text {bottom }}$ and $T u_{\max }$ for the short step-length case $\ell<0.24$ clearly indicate the existence of separated shear flow over the side surfaces. A clear contrast is observed for the long step-length case $\ell \geq 0.24$, although turbulence intensity gradually increases with increasing step length after the optimum step configuration. The turbulence intensity in this case is less than $10 \%$. Thus, the turbulence growth on the side surfaces is similar to that in the Blasius flow. As shown in Fig. 9, the maximum turbulence intensity varies depending on step length $(\ell)$ and measuring position $(x)$. Even though the real surface velocity profile could not be measured in the recent investigation due to the limitation of measuring devices, it is assumed that, in the case $\ell \geq$ 0.24 , the separated shear layer flow reattaches just at side surface of the obstacle as confirmed in flow visualization. Thus, the boundary layer developed on the side surface is considered to be very thin. Thus, it was concluded that the location of maximum turbulence intensity was attained coinciding with the side surface of the obstacle. Then, considering the result of maximum turbulence intensity, which is determined by an extrapolation method, the optimum step configuration suppresses the turbulence intensity to less than $10 \%$.

\subsubsection{Reynolds stress}

Figure 10 shows Reynolds stress $\overline{u v}$ distributions at the side surfaces of the stepped-nose obstacle for the fixed step height $h=0.16$ and the two step lengths of $\ell=0.08$ and 0.24 . The Reynolds stress distributions were measured at the same five stations as the other previously mentioned quantities. The measured Reynolds stress component expresses the transport of $x$-momentum trough a surface normal to the $y$-axis. Consistent with Figs. 5 and 8 , it takes a large magnitude in the separated flow regions for $\ell=0.08$ but it vanishes for $\ell=0.24$ except near the side surfaces downstream. The Reynolds stress in the case of $\ell=0.24$ be- 


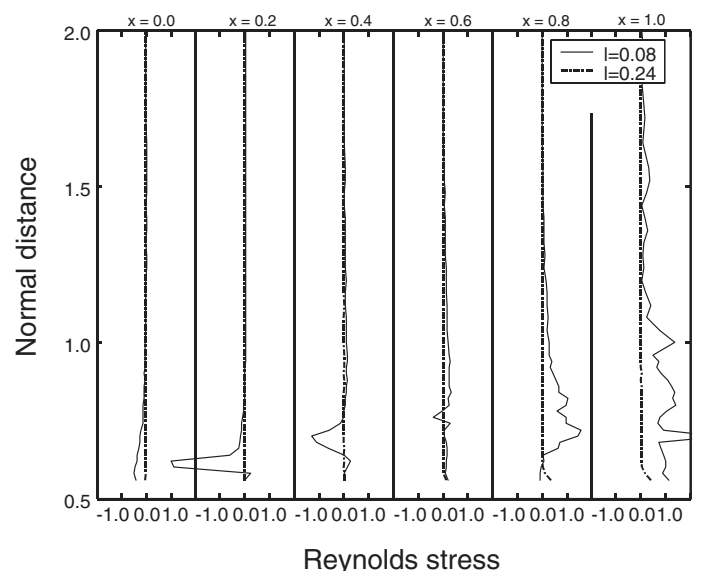

Fig. 10. Reynolds stress distribution of side flow in cases of $h=0.16$, $\ell=0.08$, and 0.24 .

comes significant near the rear edges of the side surfaces. It takes a small positive value, which monotonically decreases to zero as the normal distance from the side surface is increased. This means that the weak steady flow moving downstream is excited in the boundary layer, just like a normal vibrating plate placed parallel to a uniform stream. The situation is totally changed in the case of $\ell=0.08$. The Reynolds stress takes negative values near the leading edges of the side surfaces. Moving downstream, the minimum point moves outward consistent with the movement of the outer edge of the boundary layer, and its magnitude first increases rapidly and then decreases. This Reynolds stress distribution shows that the separated main flow is decelerated but the fluid in the separation region is accelerated downstream. This behavior is consistent with the steady streaming effect caused by oscillating flow. Toward the rear edges of the side surfaces, the Reynolds stress turns positive. This happens because of the interaction of the side flow with vortices created behind the back surface of the stepped-nose obstacle.

\subsection{Step height effect on side flow}

Fixing the step length at $\ell=0.16$, the side flow of the stepped-nose obstacle was examined for various step heights. In this series of experiments, the optimal step flow condition is realized only in the vicinity of a certain step height $(h=0.1)$, where the drag coefficient of the stepped-nose obstacle becomes minimum and the side flow of the obstacle is stabilized as seen in Figs. 11-15. Figures 11, 12 and 14, respectively, show the variations in streamwise velocity, turbulence intensity, and Reynolds stress profiles along the side surface of the stepped-nose obstacle with step heights $h=0.1$ and 0.2 . The variations in the characteristic speeds ( $U_{\text {edge }}, U_{\max }$ and $U_{\text {bottom }}$ ) and turbulence intensities ( $T u_{\text {edge }}, T u_{\text {max }}$ and $T u_{\text {bottom }}$ ) with step height are shown in Figs. 12 and 14, respectively. Trends similar to those described for Figs. 5-10 are observed in these figures.

Combining the results of various step lengths and heights, it is found that the stepped-nose method is very effective for reducing the drag coefficient when the step height is 0.1 and step length is greater than 0.24 .

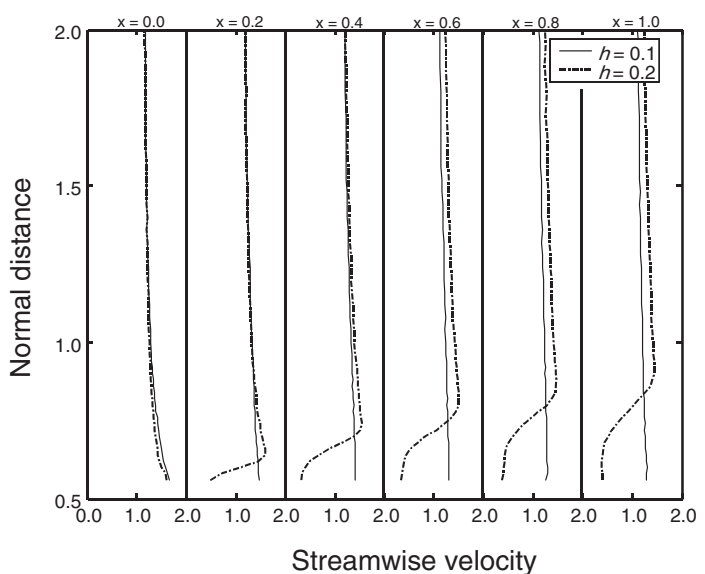

Fig. 11. Streamwise velocity distribution of side flow in cases of $\ell=$ $0.16, h=0.1$ and 0.2

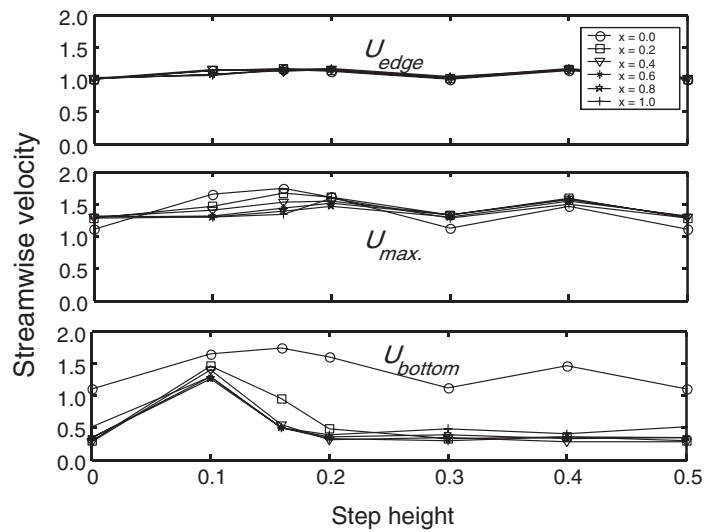

Fig. 12. Characterization of step-height dependence of time-averaged side flow for $\ell=0.16$.

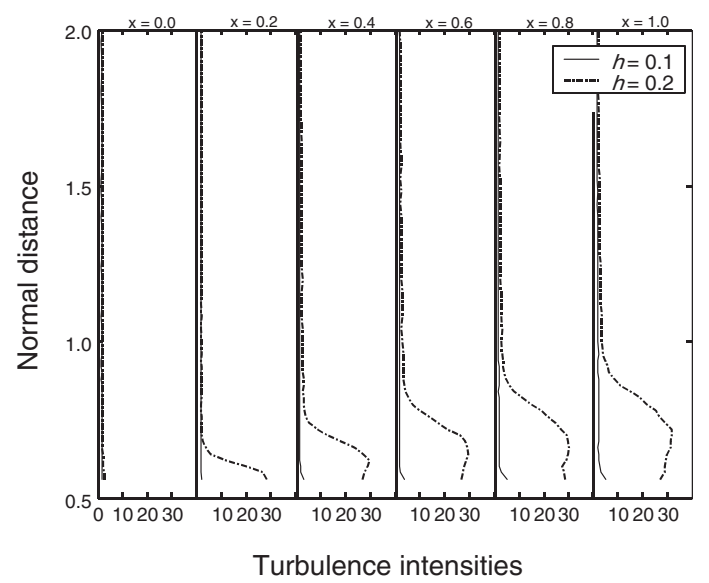

Fig. 13. Turbulence intensity distribution of side flow in cases of $\ell=$ $0.16, h=0.1$ and 0.2

\subsection{Reynolds number effect}

We examined the effects of Reynolds numbers on the flow characteristics of various step-height obstacles. The Reynolds numbers used were $5.0 \times 10^{4}, 1.0 \times 10^{5}$, and $1.5 \times 10^{5}$, obtained by increasing the free-stream velocity of the test section to $10 \mathrm{~m} / \mathrm{s}, 20 \mathrm{~m} / \mathrm{s}$, and $30 \mathrm{~m} / \mathrm{s}$, respective- 


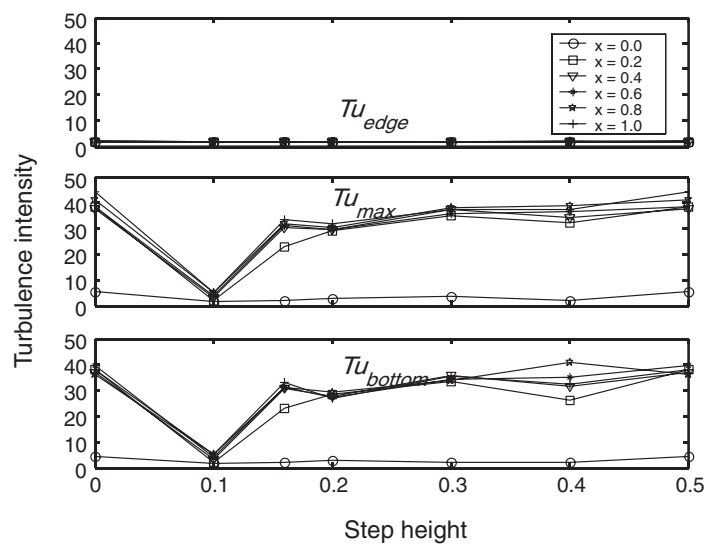

Fig. 14. Characterization of step-height dependence of turbulence intensity for $\ell=0.16$.

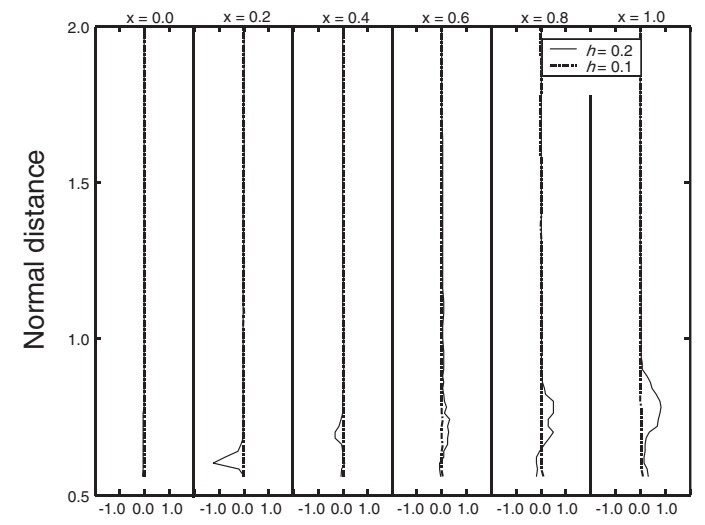

Reynolds stress

Fig. 15. Reynolds stress distribution of side flow in cases of $\ell=0.16$, $h=0.1$ and 0.2 .

ly. As shown in Figs. 16 and 17, for the tested Reynolds number range, the side flow velocity profile and turbulence intensity did not change significantly. This means that the drag reduction effect of the stepped-nose obstacle is unchanged as long as the Reynolds number is large enough. The increase in free-stream velocity accompanies the corresponding increase in free-stream turbulence. If the strength and size of the vortices trapped in the step regions are influenced by this increased free-stream turbulence, the side flow properties will change correspondingly. However, such an effect cannot be recognized for the flow properties shown in Figs. 16 and 17. Thus, we may say that the turbulence of the flow turning around the step corners, unless too strong, does not significantly affect the stepped-nose effects presented in this paper.

\section{Conclusion}

An experimental investigation of stepped-nose effects for various step lengths and heights at the forebody of a square cube has been conducted. From this investigation, we learned that there is an optimum step configuration size where the net drag force at the forebody of the stepped-nose obstacle becomes zero, the turbulence growth along the side

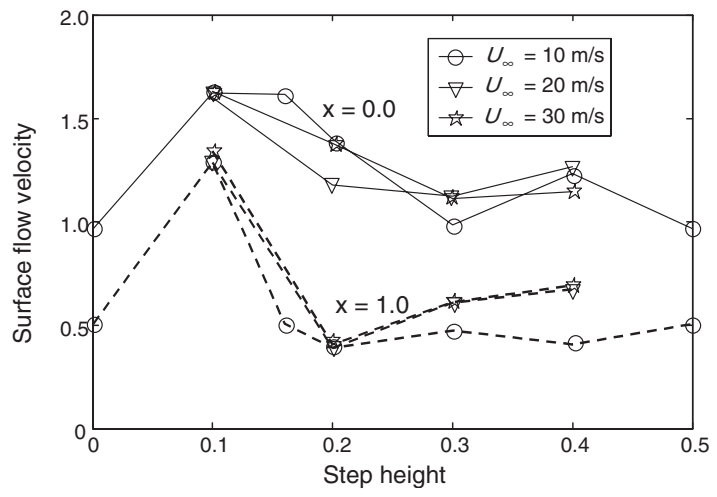

Fig. 16. Dependence of surface velocity on step height at various Reynolds numbers.

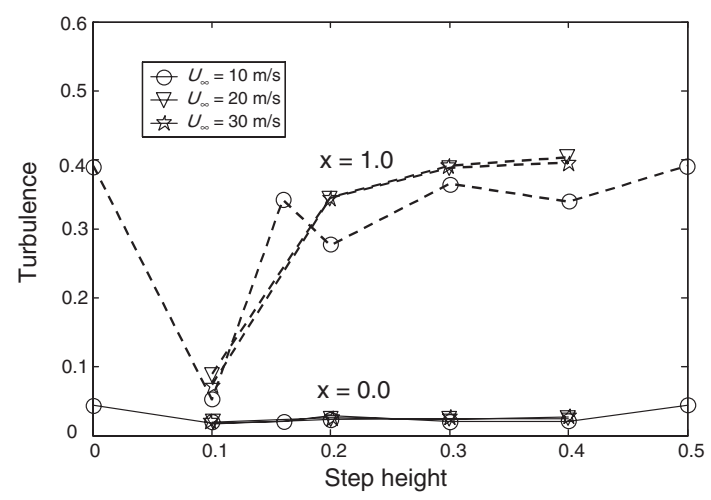

Fig. 17. Dependence of turbulence intensity on step height at various Reynolds numbers.

surface of the stepped-nose obstacle is suppressed and stabilized. Furthermore, turbulence intensity along the side surface becomes lower than $10 \%$ of the free-stream velocity. We measured the velocity distribution, turbulence, and Reynolds stress for various step lengths with a fixed step height and for various step heights with a fixed step length. We concluded that applying the optimum stepped-nose configuration, the separated flow along the side surfaces of the obstacle is affected in such a way that a stable boundary layer profile is obtained. The turbulence intensity of the optimum stepped-nose configuration could be suppressed down to $10 \%$ of the free-stream velocity. This leads to a stable Von-Karman vortex configuration, low base pressure, and large drag reduction. The pressure measurements were conducted for various step lengths. We concluded that the suction pressure acting on the step region and base pressures along the back surface of the obstacle depend on the step length. For the optimum stepped-nose configuration, pressure relaxation is observed along the side surface. This leads to a stable boundary layer and low base pressure.

\section{References}

1) Saunders, W. D.: Apparatus for Reducing Linear and Lateral Wind Resistance in a Tractor-Trailer Combination Vehicle, U.S. Patent No. 3241876, 1966.

2) Koenig, K. and Roshko, A.: An Experimental Study of Geometrical Effects on the Drag and Flow Field of Two Bluff Bodies Separated 
by a Gap, J. Fluid Mech., 156 (1985), pp. 167-204.

3) Watanabe, K.: Characteristics of Axial Flow around Step Cylinder (Parallel Flow), Trans. Jpn. Soc. Mech. Eng. B, 62 (1996), pp. 21302136 (in Japanese).

4) Cakrawala, A. and Umemura, A.: Drag Reduction, and Flow Stabilization Mechanism of Stepped-Nose Obstacle, ISTS Paper 2002-e-34, 2002.

5) Ruderich, R. and Fernholz, H. H.: An Experimental Investigation of a Turbulent Shear Flow with Separation, Reverse Flow, and Reattachment, J. Fluid Mech., 163 (1986), pp. 283-322.

6) Ota, T., Asano, T. and Okawa, J.: Reattachment Length and Transition of Separated Flow over Blunt Flat Plates, Bull. Jpn. Soc. Mech. Eng., 24 (1981), pp. 941-947.

7) Fernholz, H. H. and Vagt, J. D.: Turbulence Measurement in an Adverse Pressure Gradient Three Dimensional Turbulent Boundary Lay- er along Circular Cylinder, J. Fluid Mech., 111 (1981), pp. 233-269.

8) Cakrawala, A. and Umemura, A.: Drag Reduction and Flow Stabilization Mechanism of Step Nose Obstacle, Proceeding of the 39th Aircraft Symposium, 2001, pp. 87-90.

9) Lee, B. E.: The Effect of Turbulence on the Surface Pressure Field of a Square Prism, J. Fluid Mech., 69, Part 2 (1975), pp. 263-282.

10) Norberg, C.: Flow around Rectangular Cylinder: Pressure Forces and Wake Frequencies, J. Wind Eng. Ind. Aerodyn., 8 (1981), pp. 211222.

11) Murakami, S. and Machida, A.: On Turbulent Vortex Shedding Flow Past 2D Square Cylinder Predicted by CFD, J. Wind Eng. Ind. Aerodyn., 54/55 (1955), pp. 191-211.

12) Koutmos, P. and Mavridis, C.: A Computational Investigation of Unsteady Separated Flows, Int. J. Heat Fluid Flow, 18 (1997), pp. $297-$ 306 